\title{
Research Article \\ Exponential Decay to the Degenerate Nonlinear Coupled Beams System with Weak Damping
}

\author{
R. F. C. Lobato, ${ }^{1}$ D. C. Pereira, ${ }^{2}$ and M. L. Santos ${ }^{1}$ \\ ${ }^{1}$ Programa de Pós-Graduação, em Matemática Eestatística, Faculdade de Matemática, Universidade Federal \\ do Pará, Campus Universitário do Guamá, Rua Augusto Corrêa 01, 66075-110 Belém, PA, Brazil \\ 2 Departamento de Matemática, Estatística e Informática, Universidade do Estado do Pará, Rua do Una 156, \\ Telégrafo, 66113-200 Belém, PA, Brazil
}

Correspondence should be addressed to M. L. Santos, ls@ufpa.br

Received 10 April 2012; Accepted 3 July 2012

Academic Editors: D. Dürr and W.-H. Steeb

Copyright (C) 2012 R. F. C. Lobato et al. This is an open access article distributed under the Creative Commons Attribution License, which permits unrestricted use, distribution, and reproduction in any medium, provided the original work is properly cited.

We consider a nonlinear degenerate coupled beams system with weak damping. We show using the Nakao method that the solution of this system decays exponentially when the time tends to infinity.

\section{Introduction}

For the last several decades, various types of equations have been employed as some mathematical models describing physical, chemical, biological, and engineering systems. Among them, the mathematical models of vibrating, flexible structures have been considerably stimulated in recent years by an increasing number of questions of practical concern. Research on stabilization of distributed parameter systems has largely focused on the stabilization of dynamic models of individual structural members such as strings, membranes, and beams.

This paper is devoted to the study of the existence, uniqueness, and uniform decay rates of the energy of solution for the nonlinear degenerate coupled beams system with weak damping given by

$$
\begin{array}{ll}
K_{1}(x, t) u_{t t}+\Delta^{2} u-M\left(\|u\|^{2}+\|v\|^{2}\right) \Delta u+u_{t}=0 & \text { in } \Omega \times(0, T) \\
K_{2}(x, t) v_{t t}+\Delta^{2} v-M\left(\|u\|^{2}+\|v\|^{2}\right) \Delta v+v_{t}=0 & \text { in } \Omega \times(0, T)
\end{array}
$$




$$
\begin{gathered}
u=v=\frac{\partial u}{\partial \eta}=\frac{\partial v}{\partial \eta}=0 \quad \text { on } \Sigma \\
(u(x, 0), v(x, 0))=\left(u_{0}, v_{0}\right) \quad \text { in } \Omega, \\
\left(u_{t}(x, 0), v_{t}(x, 0)\right)=\left(u_{1}(x), v_{1}(x)\right) \text { in } \Omega,
\end{gathered}
$$

where $\Omega$ is a bounded domain of $\mathbb{R}^{n}, n \geq 1$, with smooth boundary $\Gamma, T>0$ is a real arbitrary number, and $\eta$ is the unit normal at $\Sigma=\Gamma \times(0, T)$ direct towards the exterior of $\Omega \times(0, T)$. Here $K_{i} \in C^{1}\left([0, T] ; H_{0}^{1}(\Omega) \cap L^{\infty}(\Omega)\right), i=1,2$ and $M \in C^{1}([0, \infty[)$, see Section 2 for more details.

Problems related to the system (1.1)-(1.5) are interesting not only from the point of view of PDE general theory, but also due to its applications in mechanics. For instance, when we consider only one equation without the dissipative term, that is,

$$
K(x, t) u_{t t}+\Delta^{2} u-M\left(\|u\|^{2}\right) \Delta u=0 \quad \text { in } \Omega \times(0, T)
$$

and with $K(x, t)=1$, it is a generalization of one-dimensional model proposed by Woinowsky-Krieger [1] as a model for the transverse deflection $u(x, t)$ of an extensible beam of natural length whose ends are held a fixed distance apart. The nonlinear term represents the change in the tension of the beam due to its extensibility. The model has also been discussed by Eisley [2], while related experimental results have been given by Burgreen [3]. Dickey [4] considered the initial-boundary value problem for one-dimensional case of (1.6) with $K(x, t)=1$ in the case when the ends of the beam are hinged. He showed how the model affords a description of the phenomenon of "dynamic buckling." The one-dimensional case has also been studied by Ball [5]. He extended the work of Dickey [4] in several directions. In both cases he used the techniques of Lions [6] to prove that the initial boundary value problem is weakly well-posed. Menzala [7] studied the existence and uniqueness of solutions of (1.6) with $K(x, t)=1, x \in \mathbb{R}^{n}$, and $M \in C^{1}\left[0, \infty\left[\right.\right.$ and $M(\lambda) \geq m_{0}>0$, for all $\lambda \geq 0$. The existence, uniqueness, and boundary regularity of weak solutions were considered by Ramos [8] with $K(x) \geq k_{0}>0, x \in \Omega$. See also Pereira et al. [9]. The abstract model

$$
u_{t t}+\mathbf{A}^{2} u+M\left(\left|\mathbf{A}^{1 / 2}\right|^{2}\right) \mathbf{A} u=0
$$

of (1.6), where A is a nonbounded self-adjoint operator in a conveniently Hilbert space has been studied by Medeiros [10]. He proved that the abstract model is well-posed in the weak sense, since $M \in C^{1}\left[0, \infty\right.$ [ with $M(\lambda) \geq m_{0}+m_{1} \lambda$, for all $\lambda \geq 0$, where $m_{0}$ and $m_{1}$ are positive constants. Pereira [11] considered the abstract model (1.7) with dissipative term $u_{t}$. He proved the existence, uniqueness, and exponential decay of the solutions with the following assumptions about $M$ :

$$
M \in C^{0}\left(\left[0, \infty[) \text { with } M(\lambda) \geq-\beta, \quad \forall \lambda \geq 0,0<\beta<\lambda_{1},\right.\right.
$$

where $\lambda_{1}$ is the first eigenvalue of

$$
\mathbf{A}^{2} u-\lambda \mathbf{A} u=0 .
$$


Our main goal here is to extend the previous results for a nonlinear degenerate coupled beams system of type (1.1)-(1.5). We show the existence, uniqueness, and uniform exponential decay rates.

Our paper is organized as follows. In Section 2 we give some notations and state our main result. In Section 3 we obtain the existence and uniqueness for global weak solutions. To obtain the global weak solution we use the Faedo-Galerkin method. Finally, in Section 4 we use the Nakao method (see Nakao [12]) to derive the exponential decay of the energy.

\section{Assumptions and Main Result}

In what follows we are going to use the standard notations established in Lions [6].

Let us consider the Hilbert space $L^{2}(\Omega)$ endowed with the inner product

$$
(u, v)=\int_{\Omega} u(x) v(x) d x
$$

and norm

$$
|u|=\sqrt{(u, v)}
$$

We also consider the Sobolev space $H^{1}(\Omega)$ endowed with the scalar product

$$
(u, v)_{H^{1}(\Omega)}=(u, v)+(\nabla u, \nabla v)
$$

We define the subspace of $H^{1}(\Omega)$, denoted by $H_{0}^{1}(\Omega)$. This space endowed with the norm induced by the scalar product

$$
((u, v))_{H_{0}^{1}(\Omega)}=(\nabla u, \nabla v)
$$

is a Hilbert space.

\subsection{Assumptions on the Functions $K_{i}, i=1,2$, and $M$}

To obtain the weak solution of the system (1.1)-(1.5) we consider the following hypothesis:

$K_{i} \in C^{1}\left([0, T] ; H_{0}^{1}(\Omega) \cap L^{\infty}(\Omega)\right), \quad i=1,2$,

with $K_{i}(x, t) \geq 0, \quad \forall(x, t) \in \Omega \times(0, T)$,

and there exists $\gamma>0$ such that $K_{i}(x, 0) \geq \gamma>0$,

$$
\left|\frac{\partial K_{i}}{\partial t}\right|_{\mathbb{R}} \leq \delta+C(\delta) K_{i}, \quad i=1,2, \forall \delta>0,
$$


$M \in C^{1}([0, \infty[)$ with $M(\lambda) \geq-\beta, \quad \forall \lambda \geq 0$,

$0<\beta<\lambda_{1}, \quad \lambda_{1}$ is the first eingenvalue of the stationary problem,

$$
\Delta^{2} u-\lambda(-\Delta u)=0 .
$$

Remark 2.1. Let $\lambda_{1}$ be the first eingevalue of $\Delta^{2} u-\lambda(-\Delta u)=0$; then (see Miklin [13])

$$
\lambda_{1}=\inf _{w \in H_{0}^{2}(\Omega)} \frac{|\Delta w|^{2}}{|\nabla w|^{2}}>0 .
$$

\section{Existence and Uniqueness Results}

Now, we are in a position to state our result about the existence of weak solution to the system (1.1)-(1.5).

Theorem 3.1. Let one take $\left(u_{0}, v_{0}\right) \in\left(H_{0}^{1}(\Omega) \cap H^{4}(\Omega)\right)^{2}$ and $\left(u_{1}, v_{1}\right) \in\left(H_{0}^{2}(\Omega)\right)^{2}$, and let one suppose that assumptions (2.5), (2.6) and (2.7) hold. Then, there exist unique functions $u, v$ : $[0, T] \rightarrow L^{2}(\Omega)$ in the class

$$
\begin{gathered}
(u, v) \in\left(\left(L_{\mathrm{loc}}^{\infty}(0, \infty): H_{0}^{2}(\Omega) \cap H^{4}(\Omega)\right)\right)^{2}, \\
\left(u_{t}, v_{t}\right) \in\left(\left(L_{\mathrm{loc}}^{\infty}(0, \infty): H_{0}^{2}(\Omega)\right)\right)^{2} \\
\left(u_{t t}, v_{t t}\right) \in\left(\left(L_{\mathrm{loc}}^{\infty}(0, \infty): L^{2}(\Omega)\right)\right)^{2}
\end{gathered}
$$

satisfying

$$
\begin{array}{cc}
K_{1}(x, t) u_{t t}+\Delta^{2} u-M\left(\|u\|^{2}+\|v\|^{2}\right) \Delta u+u_{t}=0 & \text { in } L_{\mathrm{loc}}^{2}\left(0, \infty ; L^{2}(\Omega)\right), \\
K_{2}(x, t) v_{t t}+\Delta^{2} v-M\left(\|u\|^{2}+\|v\|^{2}\right) \Delta v+v_{t}=0 & \text { in } L_{\mathrm{loc}}^{2}\left(0, \infty ; L^{2}(\Omega)\right), \\
(u(x, 0), v(x, 0))=\left(u_{0}(x), v_{0}(x)\right) & \text { in } \Omega, \\
\left(u_{t}(x, 0), v_{t}(x, 0)\right)=\left(u_{1}(x), v_{1}(x)\right) & \text { in } \Omega .
\end{array}
$$

Proof. Since $K_{i} \geq 0, i=1,2$, we first perturb the system (1.1)-(1.5) with the terms $\varepsilon u_{t t}, \varepsilon v_{t t}$, with $0<\varepsilon<1$, and we apply the Faedo-Galerkin method to the perturbed system. After we pass to the limit with $\varepsilon \rightarrow 0$ in the perturbed system and we obtain the solution for the system (1.1)-(1.5).

(1) Perturbed System

Consider the perturbed system

$$
\begin{array}{ll}
\left(K_{1}+\varepsilon\right) u_{t t}^{\varepsilon}+\Delta u^{\varepsilon}+M\left(\left\|u^{\varepsilon}\right\|^{2}+\left\|v^{\varepsilon}\right\|^{2}\right)\left(-\Delta u^{\varepsilon}\right)+u_{t}^{\varepsilon}=0 & \text { in } \Omega \times(0, T), \\
\left(K_{2}+\varepsilon\right) v_{t t}^{\varepsilon}+\Delta v^{\varepsilon}+M\left(\left\|u^{\varepsilon}\right\|^{2}+\left\|v^{\varepsilon}\right\|^{2}\right)\left(-\Delta v^{\varepsilon}\right)+v_{t}^{\varepsilon}=0 & \text { in } \Omega \times(0, T),
\end{array}
$$


ISRN Mathematical Physics

$$
\begin{gathered}
u^{\varepsilon}=v^{\varepsilon}=\frac{\partial u^{\varepsilon}}{\partial \eta}=\frac{\partial v^{\varepsilon}}{\partial \eta}=0 \quad \text { on } \Sigma, \\
\left(u^{\varepsilon}(x, 0), v^{\varepsilon}(x, 0)\right)=\left(u_{0}(x), v_{0}(x)\right) \text { in } \Omega, \\
\left(u_{t}^{\varepsilon}(x, 0), v_{t}^{\varepsilon}(x, 0)\right)=\left(u_{1}(x), v_{1}(x)\right) \text { in } \Omega .
\end{gathered}
$$

Let $\left(w_{v}\right)_{v \in \mathbb{N}}$ be a basis of $H_{0}^{2}(\Omega)$ formed by the eigenvectors of the operator $-\Delta$, that is, $-\Delta w_{v}=\lambda_{v} w_{v}$, with $\lambda_{v} \rightarrow \infty$ when $v \rightarrow \infty$. Let $V_{m}=\left[w_{1}, w_{2}, \ldots, w_{m}\right]$ be the subspace generated by the first $m$ vectors of $\left(w_{v}\right)_{v \in \mathbb{N}}$.

For each fixed $\varepsilon$, we consider

$$
\begin{aligned}
& u_{m}^{\varepsilon}(t)=\sum_{j=1}^{m} g_{j \varepsilon m}(t) w_{j} \in V_{m}, \\
& v_{m}^{\varepsilon}(t)=\sum_{j=1}^{m} h_{j \varepsilon m}(t) w_{j} \in V_{m}
\end{aligned}
$$

as solutions of the approximated perturbed system

$$
\begin{gathered}
\left(\left(K_{1}+\varepsilon\right) u_{t t m}^{\varepsilon}(t), w\right)+\left(-\Delta u_{m}^{\varepsilon}(t),-\Delta w\right) \\
+M\left(\left\|u_{m}^{\varepsilon}(t)\right\|^{2}+\left\|v_{m}^{\varepsilon}(t)\right\|^{2}\right)\left(-\Delta u_{m}^{\varepsilon}(t), w\right)+\left(u_{t m}^{\varepsilon}(t), w\right)=0, \quad \forall w \in V_{m} \\
\left(\left(K_{2}+\varepsilon\right) v_{t t m}^{\varepsilon}(t), z\right)+\left(-\Delta v_{m}^{\varepsilon}(t),-\Delta z\right) \\
+M\left(\left\|u_{m}^{\varepsilon}(t)\right\|^{2}+\left\|v_{m}^{\varepsilon}(t)\right\|^{2}\right)\left(-\Delta v_{m}^{\varepsilon}(t), z\right)+\left(v_{t m}^{\varepsilon}(t), z\right)=0, \quad \forall z \in V_{m} \\
\left(u_{m}^{\varepsilon}(0), v_{m}^{\varepsilon}(0)\right)=\left(u_{0 m}, v_{0 m}\right) \longrightarrow\left(u_{0}, v_{0}\right) \quad \text { in }\left(H_{0}^{2}(\Omega) \cap H^{4}(\Omega)\right)^{2} \\
\left(u_{t m}^{\varepsilon}(0), v_{t m}^{\varepsilon}(0)\right)=\left(u_{1 m}, v_{1 m}\right) \longrightarrow\left(u_{1}, v_{1}\right) \text { in }\left(H_{0}^{2}(\Omega)\right)^{2}
\end{gathered}
$$

The local existence of the approximated solutions $\left(u_{m}^{\varepsilon}, v_{m}^{\varepsilon}\right)$ is guaranteed by the standard results of ordinary differential equations. The extension of the solutions $\left(u_{m}^{\varepsilon}, v_{m}^{\varepsilon}\right)$ to the whole interval $[0, T]$ is a consequence of the first estimate below.

\section{The First Estimate}

Setting $w=u_{t m}^{\varepsilon}$ and $z=v_{t m}^{\varepsilon}$ in (3.6) and (3.7), respectively, integrating over (0,t), and taking the convergences (3.8) and (3.9) in consideration, we arrive at

$$
\begin{aligned}
& \left(K_{1},\left(u_{t m}^{\varepsilon}\right)^{2}(t)\right)+\varepsilon\left|u_{t m}^{\varepsilon}(t)\right|^{2}+\left|\Delta u_{m}^{\varepsilon}(t)\right|^{2}+\left(K_{2},\left(v_{t m}^{\varepsilon}\right)^{2}(t)\right)+\varepsilon\left|v_{t m}^{\varepsilon}\right|^{2} \\
& \quad+\left|\Delta v_{m}^{\varepsilon}(t)\right|^{2}+\widehat{M}\left(\left\|u_{m}^{\varepsilon}(t)\right\|^{2}+\left\|v_{m}^{\varepsilon}(t)\right\|^{2}\right)+2 \int_{0}^{t}\left[\left|u_{t m}^{\varepsilon}(s)\right|^{2}+\left|v_{t m}^{\varepsilon}(s)\right|^{2}\right] d s
\end{aligned}
$$




$$
\begin{aligned}
\leq & \int_{0}^{t}\left[\left|\left(\frac{\partial K_{1}}{\partial t},\left(u_{t m}^{\varepsilon}\right)^{2}(s)\right)\right|_{\mathbb{R}}+\left|\left(\frac{\partial K_{2}}{\partial t},\left(v_{t m}^{\varepsilon}\right)^{2}(s)\right)\right|_{\mathbb{R}}\right] d s+\left(K_{1}(0), u_{1 m}^{2}\right)+\varepsilon\left|u_{1 m}\right|^{2} \\
& +\left|\Delta u_{0 m}\right|^{2}+\left(K_{2}(0), v_{1 m}^{2}\right)+\varepsilon\left|v_{1 m}\right|^{2}+\left|\Delta v_{0 m}\right|^{2}+\widehat{M}\left(\left\|u_{0 m}\right\|^{2}+\left\|v_{0 m}\right\|^{2}\right)
\end{aligned}
$$

where

$$
\widehat{M}(s)=\int_{0}^{s} M(\tau) d \tau
$$

From (2.7) and (2.8), we have

$$
\widehat{M}\left(\left\|u_{m}^{\varepsilon}(t)\right\|^{2}+\left\|v_{m}^{\varepsilon}(t)\right\|^{2}\right) \geq-\frac{\beta}{\lambda_{1}}\left(\left|\Delta u_{m}^{\varepsilon}(t)\right|^{2}+\left|\Delta v_{m}^{\varepsilon}(t)\right|^{2}\right) .
$$

Since $\beta<\lambda_{1}$ and so by (2.5)-(2.7) and convergences (3.8), (3.9), and (3.12), we obtain

$$
\begin{aligned}
\left(K_{1},\left(u_{t m}^{\varepsilon}\right)^{2}(t)\right)+\left(K_{2},\left(u_{t m}^{\varepsilon}\right)^{2}(t)\right)+\varepsilon\left(\left|u_{t m}^{\varepsilon}(t)\right|^{2}+\left|v_{t m}^{\varepsilon}\right|^{2}(t)\right) & \\
& +\left(1-\frac{\beta}{\lambda_{1}}\right)\left(\left|\Delta u_{m}^{\varepsilon}(t)\right|^{2}+\left|\Delta v_{m}^{\varepsilon}(t)\right|^{2}\right)+(2-\delta) \int_{0}^{t}\left[\left|u_{t m}^{\varepsilon}(s)\right|^{2}+\left|u_{t m}^{\varepsilon}(s)\right|^{2}\right] d s \\
\leq & C_{0}+C(\delta) \int_{0}^{t}\left[\left(K_{1},\left(u_{t m}^{\varepsilon}\right)^{2}(s)\right)+\left(K_{2},\left(v_{t m}^{\varepsilon}\right)^{2}(s)\right)\right] d s
\end{aligned}
$$

with $0<\delta<1$ and $C_{0}$ being a positive constant independent of $\varepsilon, m$, and $t$.

Employing Gronwall's lemma in (3.13), we obtain the first estimate

$$
\begin{aligned}
& \left(K_{1},\left(u_{t m}^{\varepsilon}\right)^{2}(t)\right)+\left(K_{2},\left(u_{t m}^{\varepsilon}\right)^{2}(t)\right)+\varepsilon\left(\left|u_{t m}^{\varepsilon}(t)\right|^{2}+\left|v_{t m}^{\varepsilon}\right|^{2}(t)\right) \\
& \quad+\left(1-\frac{\beta}{\lambda_{1}}\right)\left(\left|\Delta u_{m}^{\varepsilon}(t)\right|^{2}+\left|\Delta v_{m}^{\varepsilon}(t)\right|^{2}\right)+(2-\delta) \int_{0}^{t}\left[\left|u_{t m}^{\varepsilon}(s)\right|^{2}+\left|u_{t m}^{\varepsilon}(s)\right|^{2}\right] d s \leq C_{1},
\end{aligned}
$$

where $C_{1}$ is a positive constant independent of $\varepsilon, m$, and $t$. Then, we can conclude that

$$
\begin{gathered}
\left(K_{1}^{1 / 2} u_{t m}^{\varepsilon}\right),\left(K_{2}^{1 / 2} v_{t m}^{\varepsilon}\right) \text { are bounded in } L^{\infty}\left(0, T ; L^{2}(\Omega)\right), \\
\left(\sqrt{\varepsilon} u_{t m}^{\varepsilon}\right),\left(\sqrt{\varepsilon} v_{t m}^{\varepsilon}\right) \text { are bounded in } L^{\infty}\left(0, T ; L^{2}(\Omega)\right) \\
\left(u_{m}^{\varepsilon}\right),\left(v_{m}^{\varepsilon}\right) \text { are bounded in } L^{\infty}\left(0, T ; H_{0}^{2}(\Omega)\right) \\
\left(u_{t m}^{\varepsilon}\right),\left(v_{t m}^{\varepsilon}\right) \text { are bounded in } L^{2}\left(0, T ; L^{2}(\Omega)\right)
\end{gathered}
$$


The Second Estimate

Substituting $w=-\Delta u_{t m}^{\varepsilon}(t)$ and $z=-\Delta v_{t m}^{\varepsilon}(t)$ in (3.6) and (3.7), respectively, it holds that

$$
\begin{aligned}
\frac{d}{d t}[ & \left(\left(K_{1},\left(u_{t m}^{\varepsilon}\right)^{2}(t)\right)\right)+\left(\left(K_{2},\left(v_{t m}^{\varepsilon}\right)^{2}(t)\right)\right)+\varepsilon\left(\left\|u_{t m}^{\varepsilon}(t)\right\|^{2}+\left\|v_{t m}^{\varepsilon}(t)\right\|^{2}\right) \\
+ & \left.\left\|\Delta u_{m}^{\varepsilon}(t)\right\|^{2}+\left\|\Delta v_{m}^{\varepsilon}(t)\right\|^{2}\right]+2\left(\left\|u_{t m}^{\varepsilon}(t)\right\|^{2}+\left\|v_{t m}^{\varepsilon}(t)\right\|^{2}\right) \\
= & \left(\left(\frac{\partial K_{1}}{\partial t},\left(u_{t m}^{\varepsilon}\right)^{2}(t)\right)\right)+\left(\left(\frac{\partial K_{2}}{\partial t},\left(v_{t m}^{\varepsilon}\right)^{2}(t)\right)\right) \\
& -2 M\left(\left\|u_{m}^{\varepsilon}(t)\right\|^{2}+\left\|v_{m}^{\varepsilon}(t)\right\|^{2}\right) \cdot\left[\left(\left(-\Delta u_{m}^{\varepsilon}(t), u_{t m}^{\varepsilon}(t)\right)\right)+\left(\left(-\Delta v_{m}^{\varepsilon}(t), v_{t m}^{\varepsilon}(t)\right)\right)\right]
\end{aligned}
$$

Integrating (3.16) over $(0, t), 0<t<T$, and taking (2.5)-(2.7) and (3.8), (3.9), and first estimate into account, we infer

$$
\begin{gathered}
\left(\left(K_{1},\left(u_{t m}^{\varepsilon}\right)^{2}(t)\right)\right)+\left(\left(K_{2},\left(v_{t m}^{\varepsilon}\right)^{2}(t)\right)\right)+\varepsilon\left(\left\|u_{t m}^{\varepsilon}(t)\right\|^{2}+\left\|v_{t m}^{\varepsilon}(t)\right\|^{2}\right) \\
+\left\|\Delta u_{m}^{\varepsilon}(t)\right\|^{2}+\left\|\Delta v_{m}^{\varepsilon}(t)\right\|^{2}+2\left(\left\|u_{t m}^{\varepsilon}(t)\right\|^{2}+\left\|v_{t m}^{\varepsilon}(t)\right\|^{2}\right) \\
\quad+(2-2 \delta) \int_{0}^{t}\left(\left\|u_{t m}^{\varepsilon}(t)\right\|^{2}+\left\|v_{t m}^{\varepsilon}(t)\right\|^{2}\right) d s \leq C_{2}
\end{gathered}
$$

where $C_{2}$ is a positive constant independent of $\varepsilon, m$, and $t$. From the above estimate we conclude that

$$
\begin{gathered}
\left(K_{1}^{1 / 2} u_{t m}^{\varepsilon}\right),\left(K_{2}^{1 / 2} v_{t m}^{\varepsilon}\right) \text { are bounded in } L^{\infty}\left(0, T ; H_{0}^{1}(\Omega)\right) \\
\left(\sqrt{\varepsilon} u_{t m}^{\varepsilon}\right),\left(\sqrt{\varepsilon} v_{t m}^{\varepsilon}\right) \text { are bounded in } L^{\infty}\left(0, T ; H_{0}^{1}(\Omega)\right) \\
\left(u_{m}^{\varepsilon}\right),\left(v_{m}^{\varepsilon}\right) \text { are bounded in } L^{\infty}\left(0, T ; H_{0}^{2}(\Omega) \cap H^{4}(\Omega)\right) \\
\left(u_{t m}^{\varepsilon}\right),\left(v_{t m}^{\varepsilon}\right) \text { are bounded in } L^{2}\left(0, T ; H_{0}^{1}(\Omega)\right)
\end{gathered}
$$

The Third Estimate

Differentiating (3.6) and (3.7) with respect to $t$ and setting $w=u_{t t m}^{\varepsilon}$ and $v_{t t m^{\prime}}^{\varepsilon}$, respectively, we arrive at

$$
\begin{aligned}
& \frac{d}{d t}\left[K_{1},\left(u_{t t m}^{\varepsilon}(t)\right)^{2}+\left(K_{2},\left(v_{t t m}^{\varepsilon}(t)\right)^{2}\right)+\varepsilon\left(\left|u_{t t m}^{\varepsilon}(t)\right|^{2}+\left|v_{t t m}^{\varepsilon}\right|^{2}\right)+\left|\Delta u_{t m}^{\varepsilon}(t)\right|^{2}+\left|\Delta v_{t m}^{\varepsilon}(t)\right|^{2}\right] \\
& \quad+2\left(\left|u_{t m}^{\varepsilon}(s)\right|^{2}+\left|v_{t m}^{\varepsilon}(s)\right|^{2}\right)
\end{aligned}
$$




$$
\begin{aligned}
= & -2 M\left(\left\|u_{m}^{\varepsilon}(t)\right\|^{2}+\left\|v_{m}^{\varepsilon}(t)\right\|^{2}\right) \cdot\left[\left(-\Delta u_{t m}^{\varepsilon}(t), u_{t t m}^{\varepsilon}(t)\right)+\left(-\Delta v_{t m}^{\varepsilon}(t), v_{t t m}^{\varepsilon}(t)\right)\right] \\
& -4\left[\left(u_{m}^{\varepsilon}(\mathrm{t}),-\Delta u_{t m}^{\varepsilon}(t)\right)+\left(v_{m}^{\varepsilon}(t),-\Delta v_{t m}^{\varepsilon}(t)\right)\right] \cdot M^{\prime}\left(\left\|u_{m}^{\varepsilon}(t)\right\|^{2}+\left\|v_{m}^{\varepsilon}(t)\right\|^{2}\right) \\
& \cdot\left[\left(-\Delta u_{t m}^{\varepsilon}(t), u_{t t m}^{\varepsilon}(t)\right)+\left(-\Delta v_{t m}^{\varepsilon}(t), v_{t t m}^{\varepsilon}(t)\right)\right]+\left(\frac{\partial K_{1}}{\partial t},\left(u_{t t m}^{\varepsilon}\right)^{2}(t)\right)+\left(\frac{\partial K_{2}}{\partial t},\left(v_{t t m}^{\varepsilon}\right)^{2}(t)\right) .
\end{aligned}
$$

Integrating (3.19) over $(0, t)$, and using (2.5), (3.8), (3.9), and the norms $\left|u_{t t m}^{\varepsilon}(0)\right|^{2} \leq C_{3}$ and $\left|v_{t t m}^{\varepsilon}(0)\right|^{2} \leq C_{4}$ after employing Gronwall's lemma, we obtain the third estimate

$$
\begin{aligned}
& \left(K_{1},\left(u_{t t m}^{\varepsilon}\right)^{2}(t)\right)+\left(K_{2},\left(v_{t t m}^{\varepsilon}\right)^{2}(t)\right)+\varepsilon\left(\left|u_{t t m}^{\varepsilon}(t)\right|^{2}+\left|v_{t t m}^{\varepsilon}(t)\right|^{2}\right) \\
& \left.\quad+\left|\Delta u_{t m}^{\varepsilon}(t)\right|^{2}+\left|\Delta v_{t m}^{\varepsilon}(t)\right|^{2}\right]+(2-2 \delta) \int_{0}^{t}\left(\left|u_{t t m}^{\varepsilon}(t)\right|^{2}+\left|v_{t t m}^{\varepsilon}(t)\right|^{2}\right) d s \leq C_{5}
\end{aligned}
$$

where $C_{5}$ is a positive constant independent of $\varepsilon, m$, and $t$. From the above estimate we conclude that

$$
\begin{gathered}
\left(K_{1}^{1 / 2} u_{t t m}^{\varepsilon}\right),\left(K_{2}^{1 / 2} v_{t t m}^{\varepsilon}\right) \text { are bounded in } L^{\infty}\left(0, T ; L^{2}(\Omega)\right), \\
\left(\sqrt{\varepsilon} u_{t t m}^{\varepsilon}\right),\left(\sqrt{\varepsilon} v_{t t m}^{\varepsilon}\right) \text { are bounded in } L^{\infty}\left(0, T ; L^{2}(\Omega)\right), \\
\left(u_{t m}^{\varepsilon}\right),\left(v_{t m}^{\varepsilon}\right) \text { are bounded in } L^{\infty}\left(0, T ; H_{0}^{2}(\Omega)\right), \\
\left(u_{t t m}^{\varepsilon}\right),\left(v_{t t m}^{\varepsilon}\right) \text { are bounded in } L^{2}\left(0, T ; L^{2}(\Omega)\right) .
\end{gathered}
$$

\section{(2) Limits of Approximated Solutions}

From the Aubin-Lions theorem (see [6]) we deduce that there exist subsequences of $\left(\mathcal{u}_{m}^{\varepsilon}\right)_{m \in \mathbb{N}}$ and $\left(v_{m}^{\varepsilon}\right)_{m \in \mathbb{N}}$ such that

$$
\begin{aligned}
& u_{m}^{\varepsilon} \longrightarrow u^{\varepsilon} \quad \text { strongly in } L^{2}\left(0, T ; H_{0}^{1}(\Omega)\right), \\
& v_{m}^{\varepsilon} \longrightarrow v^{\varepsilon} \quad \text { strongly in } L^{2}\left(0, T ; H_{0}^{1}(\Omega)\right),
\end{aligned}
$$

and since $M$ is continuous, it follows that

$$
M\left(\left\|u_{m}^{\varepsilon}(t)\right\|^{2}+\left\|v_{m}^{\varepsilon}(t)\right\|^{2}\right) \longrightarrow M\left(\left\|u^{\varepsilon}(t)\right\|^{2}+\left\|v^{\varepsilon}(t)\right\|^{2}\right) .
$$


From the above estimate we can conclude that there exist subsequences of $\left(u_{m}^{\varepsilon}\right)_{m \in \mathbb{N}}$ and $\left(v_{m}^{\varepsilon}\right)_{m \in \mathbb{N}}$, that we denote also by $\left(u_{m}^{\varepsilon}\right)_{m \in \mathbb{N}}$ and $\left(v_{m}^{\varepsilon}\right)_{m \in \mathbb{N}}$ such that as $m \rightarrow \infty$ and $\varepsilon \rightarrow 0$ we have

$$
\begin{aligned}
& u_{m}^{\varepsilon} \longrightarrow u, \quad v_{m}^{\varepsilon} \longrightarrow v \quad \text { weak star in } L^{\infty}\left(0, T ; H_{0}^{2}(\Omega) \cap H^{4}(\Omega)\right), \\
& u_{t m}^{\varepsilon} \longrightarrow u_{t}, \quad v_{t m}^{\varepsilon} \longrightarrow v_{t} \quad \text { weak star } L^{\infty}\left(0, T ; H_{0}^{2}(\Omega)\right), \\
& u_{t t m}^{\varepsilon} \longrightarrow u_{t t,} \quad v_{t t m}^{\varepsilon} \longrightarrow v_{t t} \quad \text { weak star } L^{2}\left(0, T ; L^{2}(\Omega)\right), \\
& \Delta u_{m}^{\varepsilon} \longrightarrow \Delta u, \quad \Delta v_{m}^{\varepsilon} \longrightarrow \Delta v \quad \text { weak star in } L^{\infty}\left(0, T ; L^{2}(\Omega)\right), \\
& K_{1} u_{t t m}^{\varepsilon} \longrightarrow K_{1} u_{t t}, \quad K_{2} v_{t t m}^{\varepsilon} \longrightarrow K_{2} v_{t t} \quad \text { weak star in } L^{\infty}\left(0, T ; L^{2}(\Omega)\right), \\
& \Delta^{2} u_{m}^{\varepsilon} \longrightarrow \Delta^{2} u, \quad \Delta^{2} v_{m}^{\varepsilon} \longrightarrow \Delta^{2} v \quad \text { weak star in } L^{2}\left(0, T ; L^{2}(\Omega)\right), \\
& \sqrt{\varepsilon} u_{t t m}^{\varepsilon} \longrightarrow \sqrt{\varepsilon} u_{t t}, \quad \sqrt{\varepsilon} v_{t t m}^{\varepsilon} \longrightarrow \sqrt{\varepsilon} v_{t t} \quad \text { weak star in } L^{\infty}\left(0, T ; L^{2}(\Omega)\right), \\
& M\left(\left\|u_{m}^{\varepsilon}(t)\right\|^{2}+\left\|v_{m}^{\varepsilon}(t)\right\|^{2}\right)\left(-\Delta u_{m}^{\varepsilon}-\Delta v_{m}^{\varepsilon}\right)
\end{aligned}
$$

Now, multiplying (3.6), (3.7) by $\theta \in \Phi(0, T)$ and integrating over $(0, T)$, we arrive at

$$
\begin{aligned}
\int_{0}^{T}\left(\left(K_{1}+\varepsilon\right) u_{t t m}^{\varepsilon}(t), w\right) \theta(t) d t+\int_{0}^{T}\left(-\Delta u_{m}^{\varepsilon}(t),-\Delta w\right) \theta(t) d t \\
\quad+\int_{0}^{T} M\left(\left\|u_{m}^{\varepsilon}(t)\right\|^{2}+\left\|v_{m}^{\varepsilon}(t)\right\|^{2}\right)\left(-\Delta u_{m}^{\varepsilon}(t), w\right) \theta d t \\
\quad+\int_{0}^{T}\left(u_{t m}^{\varepsilon}, w\right) \theta d t=0, \quad \forall w \in V_{m}, \forall \theta \in \Phi(0, T), \\
\int_{0}^{T}\left(\left(K_{2}+\varepsilon\right) v_{t t m}^{\varepsilon}(t), z\right) \theta(t) d t+\int_{0}^{T}\left(-\Delta v_{m}^{\varepsilon}(t),-\Delta z\right) \theta(t) d t \\
\quad+\int_{0}^{T} M\left(\left\|u_{m}^{\varepsilon}(t)\right\|^{2}+\left\|v_{m}^{\varepsilon}(t)\right\|^{2}\right)\left(-\Delta v_{m}^{\varepsilon}(t), z\right) \theta d t \\
\quad+\int_{0}^{T}\left(v_{t m}^{\varepsilon}, z\right) \theta d t=0, \quad \forall z \in V_{m}, \forall \theta \in \Phi(0, T) .
\end{aligned}
$$

The convergences (3.24) are sufficient to pass to the limit in (3.25) in order to obtain

$$
\begin{array}{ll}
K_{1} u_{t t}+\Delta^{2} u+M\left(\|u(t)\|^{2}+\|v(t)\|^{2}\right)(-\Delta u)+u_{t}=0 & \text { in } L_{\mathrm{loc}}^{\infty}\left(0, \infty ; L^{2}(\Omega)\right), \\
K_{2} v_{t t}+\Delta^{2} v+M\left(\|u(t)\|^{2}+\|v(t)\|^{2}\right)(-\Delta v)+v_{t}=0 & \text { in } L_{\mathrm{loc}}^{\infty}\left(0, \infty ; L^{2}(\Omega)\right),
\end{array}
$$

and $(u, v)$ satisfies (3.1). 
The uniqueness and initial conditions follow by using the standard arguments as in Lions [6]. The proof is now complete.

\section{Asymptotic Behavior}

In this section we study the asymptotic behavior of solutions to the system (1.1)-(1.5). We show using the Nakao method that the system (1.1)-(1.5) is exponentially stable. The main result of this paper is given by the following theorem.

Theorem 4.1. Let one take $\left(u_{0}, v_{0}\right) \in\left(H_{0}^{1}(\Omega) \cap H^{4}(\Omega)\right)^{2}$, and $\left(u_{1}, v_{1}\right) \in\left(H_{0}^{2}(\Omega)\right)^{2}$ and let one suppose that assumptions (2.5), (2.6), and (2.7) hold. Then, the solution $(u, v)$ of system (1.1)-(1.5) satisfies

$$
\left|K_{1}^{1 / 2} u_{t}(t)\right|^{2}+\left|K_{2}^{1 / 2} v_{t}(t)\right|^{2}+|\Delta u(t)|^{2}+|\Delta v(t)|^{2}+\int_{t}^{t+1}\left(\left|u_{t}(s)\right|^{2}+\left|v_{t}(s)\right|^{2}\right) d s \leq \alpha_{1} e^{-\alpha_{2} t}
$$

for all $t \geq 1$, where $\alpha_{1}$ and $\alpha_{2}$ are positive constants.

Proof. Multiplying (3.2) by $u_{t}(t)$ and $v_{t}(t)$, respectively, and integrating over $\Omega$, we obtain

$$
\begin{gathered}
\frac{1}{2} \frac{d}{d t}\left[\left|K_{1}^{1 / 2} u_{t}(t)\right|^{2}+\left|K_{2}^{1 / 2} v_{t}(t)\right|^{2}+|\Delta u(t)|^{2}+|\Delta v(t)|^{2}+\widehat{M}\left(\|u(t)\|^{2}+\|v(t)\|^{2}\right)\right] \\
+\left|u_{t}(t)\right|^{2}+\left|v_{t}(t)\right|^{2}=\left(\frac{\partial K_{1}}{\partial t}, u_{t}^{2}(t)\right)+\left(\frac{\partial K_{2}}{\partial t}, v_{t}^{2}(t)\right)
\end{gathered}
$$

where

$$
\widehat{M}(s)=\int_{0}^{s} M(\tau) d \tau
$$

Using (2.6) and considering $\delta>0$ sufficiently small, we get

$$
\begin{gathered}
\frac{1}{2} \frac{d}{d t}\left[\left|K_{1}^{1 / 2} u_{t}(t)\right|^{2}+\left|K_{2}^{1 / 2} v_{t}(t)\right|^{2}+|\Delta u(t)|^{2}+|\Delta v(t)|^{2}+\widehat{M}\left(\|u(t)\|^{2}+\|v(t)\|^{2}\right)\right] \\
+\left(1-\delta-K_{0} C(\delta)\right)\left(\left|u_{t}(t)\right|^{2}+\left|v_{t}(t)\right|^{2}\right) \leq 0
\end{gathered}
$$

where $K_{0}=\max \left\{\overline{K_{1}}, \overline{K_{2}}\right\}$ with

$$
\overline{K_{i}}=\max _{s \in[t, t+1]}\left\{\operatorname{ess} \sup K_{i}(x, s)\right\}, \quad i=1,2 .
$$

Integrating (4.4) from 0tot, we have

$$
E(t)+\left(1-\delta-K_{0} C(\delta)\right) \int_{0}^{t}\left(\left|u_{t}(s)\right|^{2}+\left|v_{t}(s)\right|^{2}\right) d s \leq E(0),
$$


where

$$
E(t)=\frac{1}{2}\left[\left|K_{1}^{1 / 2} u_{t}(t)\right|^{2}+\left|K_{2}^{1 / 2} v_{t}(t)\right|^{2}+|\Delta v(t)|^{2}+|\Delta v(t)|^{2}+\widehat{M}\left(\|u(t)\|^{2}+\|v(t)\|^{2}\right)\right]
$$

is the energy associated with the system (1.1)-(1.5). From (4.4) we conclude that

$$
\frac{d}{d t} E(t) \leq 0 \quad \forall t \in(0, \infty),
$$

that is, $E(t)$ is bounded and increasing in $(0, \infty)$.

Integrating (4.4) from $\tau_{1}$ to $\tau_{2}, 0<\tau_{1}<\tau_{2}<\infty$, we arrive at

$$
E\left(\tau_{2}\right)+\left(1-\delta-K_{0} C(\delta)\right) \int_{\tau_{1}}^{\tau_{2}}\left(\left|u_{t}(s)\right|^{2}+\left|v_{t}(s)\right|^{2}\right) d s \leq E\left(\tau_{1}\right) .
$$

Taking $\tau_{1}=t$ and $\tau_{2}=t+1$ in (4.9), we get

$$
\int_{t}^{t+1}\left(\left|u_{t}(s)\right|^{2}+\left|v_{t}(s)\right|^{2}\right) d s \leq \frac{1}{1-\delta-K_{0} C(\delta)}[E(t)-E(t+1)]=F^{2}(t) .
$$

Therefore, there exist two points $t_{1} \in[t, t+1 / 4]$ and $t_{2} \in[t+3 / 4, t+1]$, such that

$$
\left|u_{t}\left(t_{i}\right)\right|+\left|v_{t}\left(t_{i}\right)\right| \leq 4 F(t), \quad i=1,2
$$

Making the inner product in $L^{2}(\Omega)$ of (1.1) and (1.2) by $u(t)$ and $v(t)$, respectively, and summing up the result we obtain

$$
\begin{aligned}
& \frac{d}{d t}\left(K_{1} u_{t}(t), u(t)\right)+\frac{d}{d t}\left(K_{2} v_{t}(t), v(t)\right)-\left|\sqrt{K_{1}} u_{t}(t)\right|^{2}-\left|\sqrt{K_{2}} v_{t}(t)\right|^{2} \\
& +|\Delta u(t)|^{2}+|\Delta v(t)|^{2}+M\left(\|u(t)\|^{2}+\|v(t)\|^{2}\right)\left(\|u(t)\|^{2}+\|v(t)\|^{2}\right)+\left(u_{t}(t), u(t)\right) \\
& +\left(v_{t}(t), v(t)\right)=\left(\frac{\partial K_{1}}{\partial t} u_{t}(t), u(t)\right)+\left(\frac{\partial K_{2}}{\partial t} v_{t}(t), v(t)\right) .
\end{aligned}
$$

Integrating from $t_{1}$ to $t_{2}$ and using (2.6), and (2.7) we have

$$
\begin{aligned}
\left(1-\frac{\beta}{\lambda_{1}}\right) & \int_{t_{1}}^{t_{2}}\left(|\Delta u(s)|^{2}+|\Delta v(s)|^{2}\right) d s \\
\leq & \left(K_{1} u_{t}\left(t_{1}\right), u\left(t_{1}\right)\right)-\left(K_{1} u_{t}\left(t_{2}\right), u\left(t_{2}\right)\right)+\left(K_{2} v_{t}\left(t_{1}\right), v\left(t_{1}\right)\right)-\left(K_{2} v_{t}\left(t_{2}\right), v\left(t_{2}\right)\right) \\
& +\left(1+\delta+K_{0} C(\delta)\right) \int_{t_{1}}^{t_{2}}\left(\left|u_{t}(s)\right||u(s)|+\left|v_{t}(s) \| v(s)\right|\right) d s \\
& +K_{0} \int_{t_{1}}^{t_{2}}\left(\left|u_{t}(s)\right|^{2}+\left|v_{t}(s)\right|^{2}\right) d s .
\end{aligned}
$$


Let us consider $C>0$ such that

$$
|u(s)| \leq C|\Delta u(s)|, \quad|v(s)| \leq C|\Delta v(s)|
$$

and we take $d>0$ sufficiently small Then we have

$$
\begin{aligned}
(1+\delta+ & \left.K_{0} C(\delta)\right)\left(\left|u_{t}(s)\right||u(s)|+\left|v_{t}(s)\right||v(s)|\right) \\
& \leq \frac{\left(1+\delta+K_{0} C(\delta)\right)^{2}}{d}\left(\left|u_{t}(s)\right|^{2}+\left|v_{t}(s)\right|^{2}\right)+d\left(|\Delta u(s)|^{2}+|\Delta v(s)|^{2}\right) \\
\left|\left(K_{1} u_{t}\left(t_{1}\right), u\left(t_{1}\right)\right)+\left(K_{2} v_{t}\left(t_{1}\right), v\left(t_{1}\right)\right)-\left(K_{1} u_{t}\left(t_{2}\right), u\left(t_{2}\right)\right)-\left(K_{2} v_{t}\left(t_{2}\right), v\left(t_{2}\right)\right)\right| & \\
& \leq C K_{0} \text { ess } \sup _{s \in[t, t+1]}|\Delta u(s)|\left(\left|u_{t}\left(t_{1}\right)\right|+\left|u_{t}\left(t_{2}\right)\right|\right)+C K_{0} \text { ess } \sup _{s \in[t, t+1]}|\Delta v(s)|\left(\left|v_{t}\left(t_{1}\right)\right|+\left|v_{t}\left(t_{2}\right)\right|\right) .
\end{aligned}
$$

Thus, substituting (4.15) into (4.13), we arrive at

$$
\begin{aligned}
\left(1-\frac{\beta}{\lambda_{1}}\right) \int_{t_{1}}^{t_{2}}\left(|\Delta u(s)|^{2}+|\Delta v(s)|^{2}\right) d s \\
\leq K_{0} \int_{t_{1}}^{t_{2}}\left(\left|u_{t}(s)\right|^{2}+\left|v_{t}(s)\right|^{2}\right) d s+d \int_{t_{1}}^{t_{2}}\left(|\Delta u(s)|^{2}+|\Delta v(s)|^{2}\right) d s \\
\quad+C K_{0} \text { ess } \sup _{s \in[t, t+1]}|\Delta u(s)|\left(\left|u_{t}\left(t_{1}\right)\right|+\left|u_{t}\left(t_{2}\right)\right|\right)+C K_{0} \text { ess } \sup _{s \in[t, t+1]}|\Delta v(s)|\left(\left|v_{t}\left(t_{1}\right)\right|+\left|v_{t}\left(t_{2}\right)\right|\right) .
\end{aligned}
$$

Applying (4.10) and (4.11) in (4.16), we have

$$
\int_{t_{1}}^{t_{2}}\left(|\Delta u(s)|^{2}+|\Delta v(s)|^{2}\right) d s \leq C_{1}\left[F^{2}(t)+\operatorname{ess} \sup _{s \in[t, t+1]}(|\Delta u(s)|+|\Delta v(s)|) F(t)\right]=G^{2}(t),
$$

where $C_{1}$ is a positive constant independent of $t$. Therefore, from (4.10) and (4.17) we obtain

$$
\int_{t_{1}}^{t_{2}}\left(\left|u_{t}(s)\right|^{2}+\left|v_{t}(s)\right|^{2}+|\Delta u(s)|^{2}+|\Delta v(s)|^{2}\right) d s \leq F^{2}(t)+G^{2}(t) .
$$

Hence, there exists $t^{*} \in\left[t_{1}, t_{2}\right]$ such that

$$
\left|u_{t}\left(t^{*}\right)\right|^{2}+\left|v_{t}\left(t^{*}\right)\right|^{2}+\left|\Delta u\left(t^{*}\right)\right|^{2}+\left|\Delta v\left(t^{*}\right)\right|^{2} \leq 2\left[F^{2}(t)+G^{2}(t)\right] .
$$

Consequently,

$$
\widehat{M}\left(\|u(t)\|^{2}+\|v(t)\|^{2}\right) \leq C_{2}\left[F^{2}(t)+G^{2}(t)\right]
$$


where

$$
C_{2}=2 m_{0} \tilde{C}, \quad m_{0}=\max _{0 \leq s \leq\left(\left\|u\left(t^{*}\right)\right\|^{2}+\left\|v\left(t^{*}\right)\right\|^{2}\right)<\infty} M(s)
$$

and $\widetilde{C}$ is a positive constant such that $\left\|u\left(t^{*}\right)\right\|^{2} \leq \widetilde{C}\left|\Delta u\left(t^{*}\right)\right|^{2}$.

From (4.19) and (4.20), we have

$$
E\left(t^{*}\right) \leq C_{3}\left[F^{2}(t)+G^{2}(t)\right]
$$

Since $E(t)$ is increasing, we have

$$
\text { ess } \sup _{s \in[t, t+1]} E(s) \leq E\left(t^{*}\right)+\left(1+\delta+K_{0} C(\delta)\right) \int_{t_{1}}^{t_{2}}\left(\left|u_{t}(s)\right|^{2}+\left|v_{t}(s)\right|^{2}\right) d s
$$

Now, by (4.10), (4.22), and (4.23) we get

$$
E(t) \leq C_{4}[E(t)-E(t+1)]
$$

where $C_{4}$ is a positive constant. Then, by the Nakao lemma (see [12]) we conclude that

$$
E(t) \leq b_{1} e^{-\alpha_{2} t}, \quad \forall t \geq 1
$$

where $b_{1}$ and $\alpha_{2}$ are positive constants, that is,

$$
\left|\sqrt{K_{1}} u_{t}(t)\right|^{2}+\left|\sqrt{K_{2}} v_{t}(t)\right|^{2}+|\Delta u(t)|^{2}+|\Delta v(t)|^{2} \widehat{M}\left(\|u(t)\|^{2}+\|v(t)\|^{2}\right) \leq 2 b_{1} e^{-\alpha_{2} t}
$$

Using (2.7) we obtain

$$
\left|\sqrt{K_{1}} u_{t}(t)\right|^{2}+\left|\sqrt{K_{2}} v_{t}(t)\right|^{2}+|\Delta u(t)|^{2}+|\Delta v(t)|^{2} \leq \frac{2 b_{1}}{m_{1}} e^{-\alpha_{2} t},
$$

where

$$
m_{1}=1-\frac{\beta}{\lambda_{1}}>0
$$

From (4.10) we have

$$
\int_{t}^{t+1}\left(\left|u_{t}(s)\right|^{2}+\left|v_{t}(s)\right|^{2}\right) d s \leq \frac{1}{1-\delta-K_{0} C(\delta)}[E(t)-E(t+1)] \leq E(t) \leq b_{1} e^{-\alpha_{2} t}
$$


Therefore, from (4.27) and (4.29) we conclude that

$$
\begin{aligned}
& \left|\sqrt{K_{1}} u_{t}(t)\right|^{2}+\left|\sqrt{K_{2}} v_{t}(t)\right|^{2}+|\Delta u(t)|^{2}+|\Delta v(t)|^{2} \\
& \quad+\int_{t}^{t+1}\left(\left|u_{t}(s)\right|^{2}+\left|v_{t}(s)\right|^{2}\right) d s \leq \alpha_{1} e^{-\alpha_{2} t}, \quad \forall t \geq 1
\end{aligned}
$$

where $\alpha_{1}$ and $\alpha_{2}$ are positive constants. Now, the proof is complete.

\section{References}

[1] S. Woinowsky-Krieger, "The effect of an axial force on the vibration of hinged bars," Journal of Applied Mechanics, vol. 17, pp. 35-36, 1950.

[2] J. G. Eisley, "Nonlinear vibration of beams and rectangular plates," Zeitschrift für Angewandte Mathematik und Physik, vol. 15, pp. 167-175, 1964.

[3] D. Burgreen, "Free vibrations of a pin-ended column with constant distance between pin ends," Journal of Applied Mechanics, vol. 18, pp. 135-139, 1951.

[4] R. W. Dickey, "Free vibrations and dynamic buckling of the extensible beam," Journal of Mathematical Analysis and Applications, vol. 29, pp. 443-454, 1970.

[5] J. M. Ball, "Initial-boundary value problems for an extensible beam," Journal of Mathematical Analysis and Applications, vol. 42, pp. 61-90, 1973.

[6] J. L. Lions, Quelques Méethodes de Réesolution des Problèemes aux Limites non Lineaires, Dunod, Paris, France, 1969.

[7] G. P. Menzala, “On classical solutions of a quasilinear hyperbolic equation,” Nonlinear Analysis, vol. 3, no. 5, pp. 613-627, 1978.

[8] O. C. Ramos, "Regularity property for the nonlinear beam operator," Anais da Academia Brasileira de Ciências, vol. 61, no. 1, pp. 15-25, 1989.

[9] D. C. Pereira, S. D. B. Menezes, J. Ferreira, and E. A. Oliveira, "Existence, uniqueness and uniform decay for the nonlinear beam degenerate equation with weak damping," Applied Mathematics and Computation, vol. 154, no. 2, pp. 555-565, 2004.

[10] L. A. Medeiros, "On a new class of nonlinear wave equations," Journal of Mathematical Analysis and Applications, vol. 69, no. 1, pp. 252-262, 1979.

[11] D. C. Pereira, "Existence, uniqueness and asymptotic behavior for solutions of the nonlinear beam equation," Nonlinear Analysis, vol. 14, no. 8, pp. 613-623, 1990.

[12] M. Nakao, "Decay of solutions of some nonlinear evolution equations," Journal of Mathematical Analysis and Applications, vol. 60, no. 2, pp. 542-549, 1977.

[13] S. G. Miklin, Variational Methods in Mathematical Physics, Pergamon Press, Oxford, 1964. 


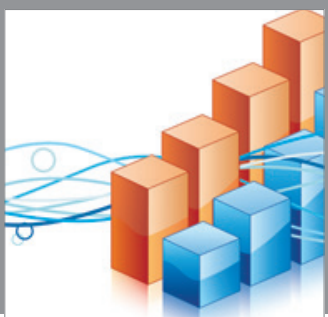

Advances in

Operations Research

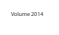

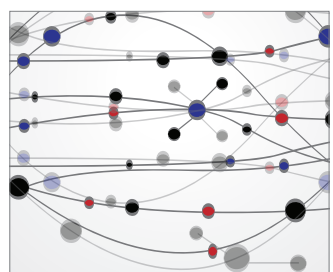

\section{The Scientific} World Journal
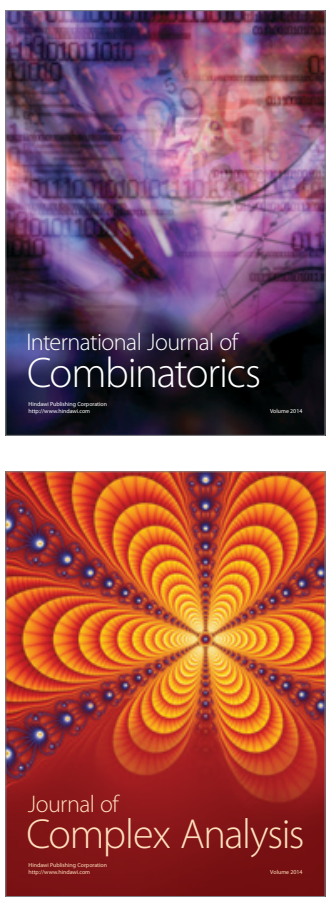

International Journal of

Mathematics and

Mathematical

Sciences
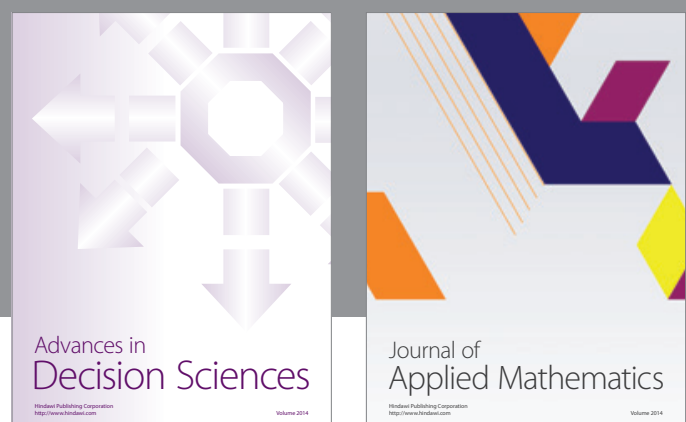

Journal of

Applied Mathematics
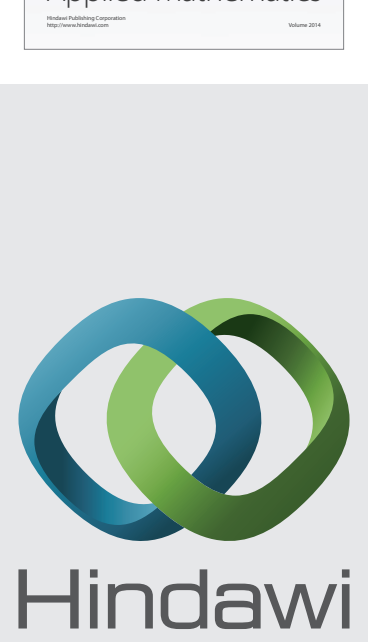

Submit your manuscripts at http://www.hindawi.com
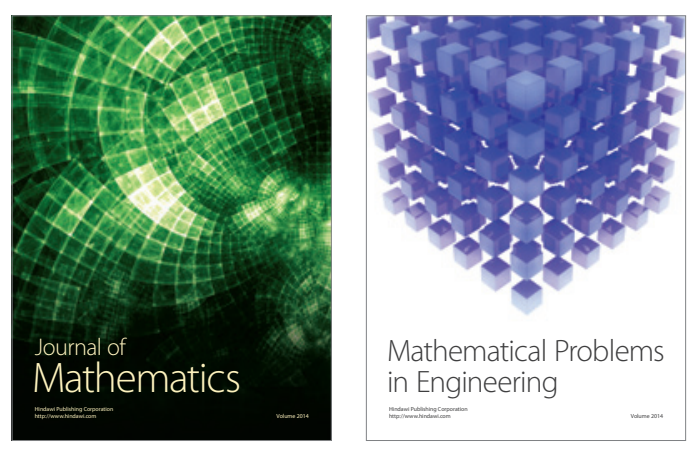

Mathematical Problems in Engineering
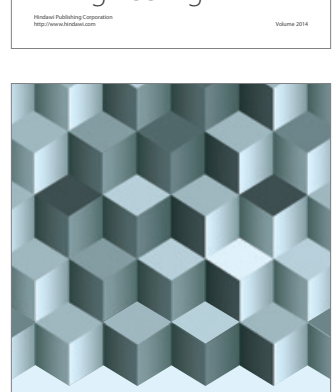

Journal of

Function Spaces
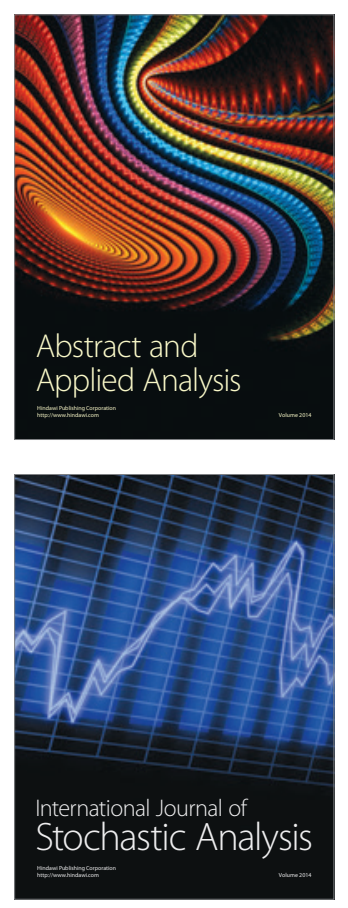

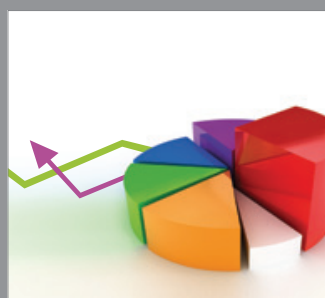

ournal of

Probability and Statistics

Promensencen
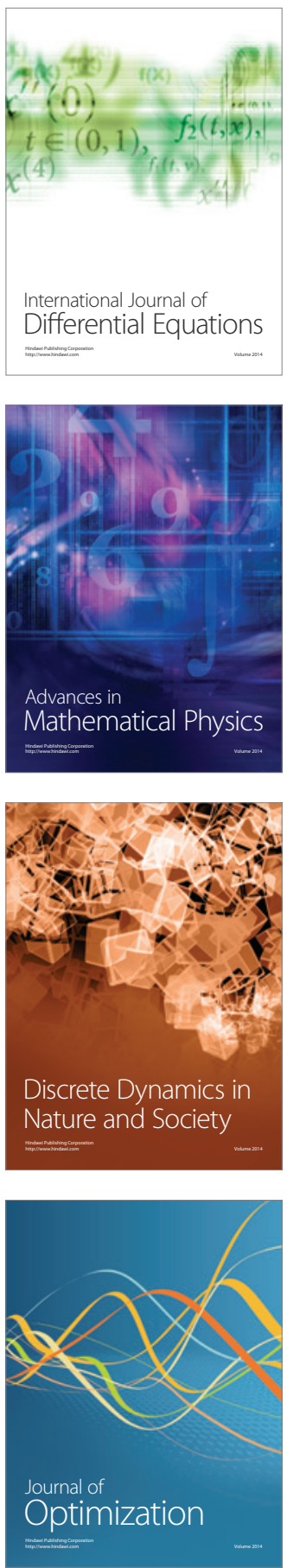